Check for updates

Cite this: RSC Adv., 2018, 8, 9272

\title{
Theoretical research on novel orthorhombic tungsten dinitride from first principles calculations
}

\begin{abstract}
Qian Li, (D)*ab Jianyun Wang ${ }^{\mathrm{b}}$ and Hanyu Liuc
Tungsten nitrides have been intensely studied for technological applications owing to their unique mechanical, chemical, and thermal properties. Combining first-principles calculations with an unbiased structural searching method (CALYPSO), we uncovered a novel orthorhombic structure with a space group $\mathrm{Cmc2}_{1}$ as the thermodynamically most stable phase for tungsten dinitride $\left(\mathrm{WN}_{2}\right)$ between $46-$ $113 \mathrm{GPa}$. The computed elastic constants and phonons reveal that the $C m c 2_{1}-W N_{2}$ structure is dynamically stable at atmospheric pressure. Moreover, hardness calculations indicate that this structure is likely to become a hard material. Our current results may stimulate further experimental work on synthesizing these technologically important materials and improve the understanding of the pressureinduced phase transitions of other transition-metal light-element compounds.
\end{abstract}

Received 4th February 2018

Accepted 24th February 2018

DOI: $10.1039 / c 8 r a 01099 d$

rsc.li/rsc-advances dinitride $\left(\mathrm{WN}_{2}\right)$ is thus expected to be a good candidate for high hardness materials, since it possessed one more $\mathrm{N}$ atom per formula unit compared to $\mathrm{WN}$.

For a long time, $\mathrm{WN}_{2}$ has been theoretically suggested to adopt $P 6_{3} / \mathrm{mmc}^{\mathbf{1 0}}$ structure at atmosphere pressure. Other researchers proposed a tetragonal phase of $\mathrm{WN}_{2}$ through replacing the $P 4 / m b m-\mathrm{ReN}_{2}$ structure. ${ }^{11}$ In this work, we established thermodynamically stable structures of $\mathrm{WN}_{2}$ over a wide range of pressures combining the first-principles calculations with unbiased structural searching methods. A novel orthorhombic $\mathrm{WN}_{2}$ with a space group $C m c 2_{1}$ is predicted which is energetically superior to the previously proposed $P 6_{3} / \mathrm{mmc}$ phase above $46 \mathrm{GPa}$ and $P 4 / \mathrm{mbm}$ phase below $113 \mathrm{GPa}$. This structure is incompressible and has not been reported before. As this newly found structure is dynamically stable, it can be prepared at high-pressure and high-temperature and quenched recovered under atmospheric conditions. This work not only revised the phase diagram of $\mathrm{WN}_{2}$ at high pressures, but also provided useful information and important guidance for the further experimental synthesis and theoretical study of $\mathrm{WN}_{2}$.

\section{Computational details}

Our global structural prediction used the unbiased intelligence based on CALYPSO ${ }^{23-25}$ methods which have successfully predicted structures of various systems. ${ }^{26-30}$ The structural relaxations, electron localization function (ELF) and electronic band structure calculations were performed using the density functional theory within the local density approximation (LDA) ${ }^{31,32}$ as implemented in the Vienna $a b$ initio simulation package (VASP) code. $^{33}$ The electron-ion interaction was described by means of projector augmented wave (PAW) ${ }^{34}$ with $5 \mathrm{~d}^{4} 6 \mathrm{~s}^{2}$ and $2 \mathrm{~s}^{2} 2 \mathrm{p}^{3}$ electrons as valence for tungsten and nitrogen atoms, 
respectively. The PAW ${ }^{34}$ method was adopted with a plane-wave kinetic-energy cutoff of $520 \mathrm{eV}$ to give excellent convergence on the total energies and structural parameters. The MonkhorstPack (MP) $k$ meshes $^{35} 0.025 \AA^{-1}$ were chosen to ensure the energies of all structures were well converged to be better than 1 meV per atom. The phonon frequencies were calculated using the direct supercell method, which uses the forces obtained by the Hellmann-Feynman theorem calculated from the optimized supercell. ${ }^{36}$ Accurate crystal elastic constants and modulus were calculated by using the Voigt-Reuss-Hill approximation. ${ }^{37}$ The simulated micro hardness ${ }^{38,39}$ were used to evaluate the mechanical properties for these $\mathrm{WN}$ compounds.

\section{Results and discussion}

In the previous theoretical studies, Wang et al. predicted a hexagonal structure with space group $P 6_{3} / m m c^{\mathbf{1 0}}$ which is potential ultra-incompressible and energetically superior to the previously proposed phase hence it is regarded as the ground state of $\mathrm{WN}_{2}$ up to $60 \mathrm{GPa}$. Yan et al. ${ }^{11}$ put forward a tetragonal phase of $\mathrm{WN}_{2}$ through replace the $P 4 / \mathrm{mbm}-\mathrm{ReN}_{2}$ structure which exhibits an unusual incompressibility along the $c$ axis on a par with diamond. This $P 4 / \mathrm{mbm}$ phase is dynamically stable, nevertheless, metastable compared to $P 6_{3} / m m c$ at $0 \mathrm{GPa} .{ }^{11}$ Due to the potential importance of this material, we have performed the systematical structural prediction for $\mathrm{WN}_{2}$ compound in the pressure range of $0-150 \mathrm{GPa}$ (3-24 atoms for each pressure) using unbiased intelligence structural searches. Here, we found that three structures could exist in different pressure ranges [Fig. 1(a)-(c)]. Besides the ground state phase $\left(P 6_{3} / m m c\right)^{10}$ and high-pressure phase $(P 4 / \mathrm{mbm})^{\mathbf{1 1}}$ which fits well with previous researches, our structural search also successfully identified a new orthorhombic structure with a space group $C m c 2_{1}$ [Fig. 1(b)] as the thermodynamically most stable phase for $\mathrm{WN}_{2}$ between 46 and $113 \mathrm{GPa}$ as shown in Fig. 1. It is noteworthy that this structure is more complex than the other two previous

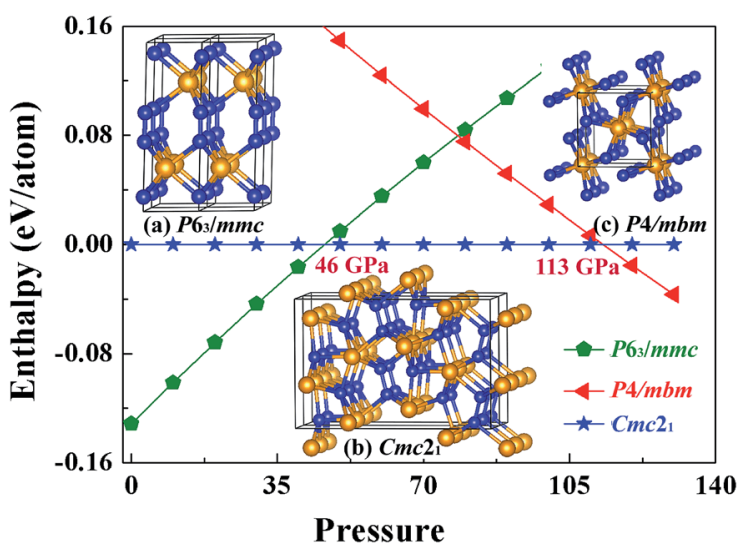

Fig. 1 Enthalpy of $\mathrm{WN}_{2}$ versus pressure. Pentagons, triangles and pentagonal stars represent $P \sigma_{3} / \mathrm{mmc}, P 4 / \mathrm{mbm}$ and $C m c 2_{1}$ structures respectively. The illustrations are the structures of the $P 6_{3} / m m c(a)$, $\mathrm{Cmc2}_{1}$ (b) and $P 4 / \mathrm{mbm}$ (c) phases of $\mathrm{WN}_{2}$. The large orange and small blue spheres represent $\mathrm{W}$ and $\mathrm{N}$ atoms, respectively. structures with higher symmetry. The present results revised the phase diagram of $\mathrm{WN}_{2}$ at high pressures.

The equilibrium lattice parameter of the $C m c 2_{1}-\mathrm{WN}_{2}$ structure are $a=2.909 \AA, b=6.875 \AA, c=10.517 \AA$ at 0 GPa while $a=$ $2.802 \AA, b=6.721 \AA, c=10.153 \AA$ at $50 \mathrm{GPa}$. There are eight $\mathrm{WN}_{2}$ formula units in the unit cell, and the tungsten atoms occupied the Wyckoff $4 d(0,0.150,0.581), 4 d(0.500,0.942,0.792)$, while the nitrogen atoms at the $8 b(0.500,0.774,0.231), 8 b(0.000$, $0.247,0.947)$ positions. The density of $C m c 2_{1}-\mathrm{WN}_{2}$ is $13.383 \mathrm{~g} \mathrm{~cm}^{-3}$ at $0 \mathrm{GPa}$. Through observation of $C m c 2_{1}-\mathrm{WN}_{2}$ along the $\mathrm{A}$ axis, we prove that the structure is arranged in a neat layer while disordered along other directions [Fig. 2(a) and (b)]. Besides, this phase consists of a fundamental building block connected by irregular rings [Fig. 2(c)]. We have selected three representative bonding modalities of nitrogen atoms in the $\mathrm{Cmc2}_{1}-\mathrm{WN}_{2}$ structure and exhibited in different crystallographic planes. The first kind of nitrogen atom has five neighboring tungsten atoms [Fig. 2(d)] with $\mathrm{W}-\mathrm{N}$ bond lengths of $2.050 \AA$, $2.175 \AA$ and $2.210 \AA$. The other two kinds of nitrogen atoms have four neighboring atoms. By contrast, one of them is adjacent to four tungsten atoms [Fig. 2(e)] while another one has a neighboring nitrogen atom [Fig. 2(f)]. The bond lengths are shown in the Fig. 2. It is worth noting that the $\mathrm{N}-\mathrm{N}$ bond length is $1.400 \AA$ at 0 GPa while $1.364 \AA$ at 50 GPa [Fig. 2(f)].

We calculated the charge density distribution and Electron Localization Function (ELF) that enables an effective and reliable analysis of the interaction between atoms. Here, we use a high isosurface value of 0.8 which indicates the possible formation of strong covalent bonds. ${ }^{\mathbf{4 0 , 4 1}}$ Previous studies have shown that an important ultra-incompressible effect comes

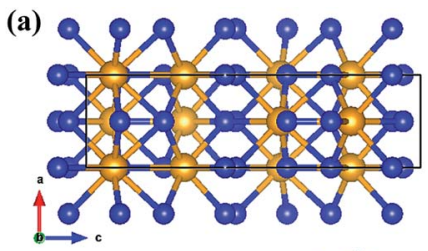

(c)

(fi)

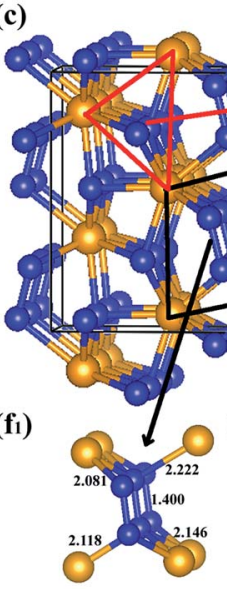

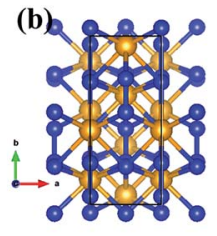

(di)

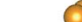

$\left(d_{2}\right)$

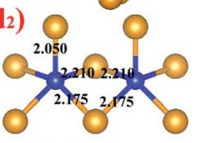

\section{.}

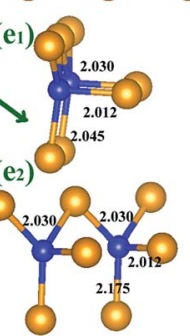

Fig. 2 The $\mathrm{Cmc2}_{1}-\mathrm{WN}_{2}$ structure is shown in different crystallographic planes in order to perceive the structures more clearly (a)-(c). The bond lengths are pinpoints as shown in (d), (e) and (f). The large orange and small blue spheres represent $\mathrm{W}$ and $\mathrm{N}$ atoms, respectively. 


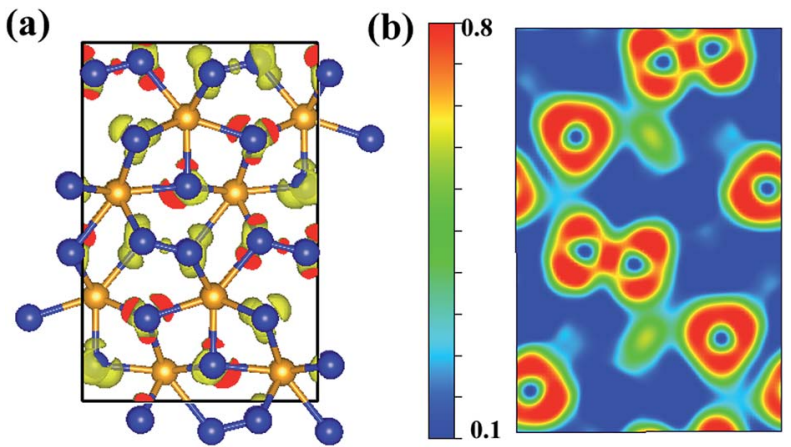

Fig. 3 Contours of the ELF of $C m c 2_{1}-W_{2}$ with isosurface $=0.8$. The $2 D-E L F$ is given for better observation.

from strong covalent $\mathrm{N}-\mathrm{N}$ bonding. ${ }^{\mathbf{2 0 , 2 1}}$ High electron localization can be clearly seen in the region between adjacent $\mathrm{N}-\mathrm{N}$ and $\mathrm{W}-\mathrm{N}$ bonds, indicative of strong covalent bonding (Fig. 3). As shown in the Fig. 3, the bonding environment of nitrogen atoms is much more complex. The calculated ELF exhibits that some nitrogen atoms in $C m c 2_{1}-\mathrm{WN}_{2}$ structure are fourfold coordinated with three near-neighbor tungsten atoms and one nitrogen atom hence forming a three-dimensional covalently bonded framework. Some nitrogen atoms are five-coordinated with neighboring tungsten atoms. The other nitrogen atoms are four-coordinated and forming a stable $\mathrm{sp}^{3}$ bonding state with tungsten atoms. These strong three-dimensional covalent bonds could reasonably lead to super hardness properties of $C m c 2_{1}-\mathrm{WN}_{2}$ structure. The calculated charge of $C m c 2_{1}-\mathrm{WN}_{2}$ showed there is the charge transfer between $\mathrm{W}(\sim 2.774)$ and $\mathrm{N}$ (1.449 and 1.327) atoms.

As the strong covalent $\mathrm{W}-\mathrm{N}$ and $\mathrm{N}-\mathrm{N}$ bonding play a key role in the ultra-incompressibility, $C m c 2_{1}-\mathrm{WN}_{2}$ may become one of the potential candidates for superhard materials. It is of the fundamental importance to compute the mechanical properties and hardness of $\mathrm{WN}_{2}$. The computed elastic constants $\left(C_{i j}\right)$ are listed in Table 1 as well as the previously predicted $P 6_{3} / m m c$ and $\mathrm{P} 4 / \mathrm{mbm}$ for comparison. It is well known that, for orthogonal structure, the mechanical stability requires that the elastic constants have to satisfy the following conditions: ${ }^{42} C_{i i}>0(i=1-$ 6), $C_{11}+C_{22}+C_{33}+2\left(C_{12}+C_{13}+C_{23}\right)>0, C_{11}+C_{22}-2 C_{12}>0, C_{11}$ $+C_{33}-2 C_{13}>0, C_{22}+C_{33}-2 C_{23}>0$. For tetragonal and hexagonal structures, the $C_{i j}$ 's need satisfy the following conditions: $C_{11}>0, C_{33}>0, C_{44}>0, C_{66}>0,\left(C_{11}-C_{12}\right)>0,\left(C_{11}+\right.$ $\left.C_{33}-2 C_{13}\right)>0,2\left(C_{11}+C_{12}\right)+C_{33}+4 C_{13}>0$ and $C_{44}>0, C_{11}>$ $\left|C_{12}\right|,\left(C_{11}+C_{12}\right) \times C_{33}>2 C_{12},{ }^{2}$ respectively. The elastic constants in Table 1 satisfy all of the conditions above, indicating they are mechanically stable.
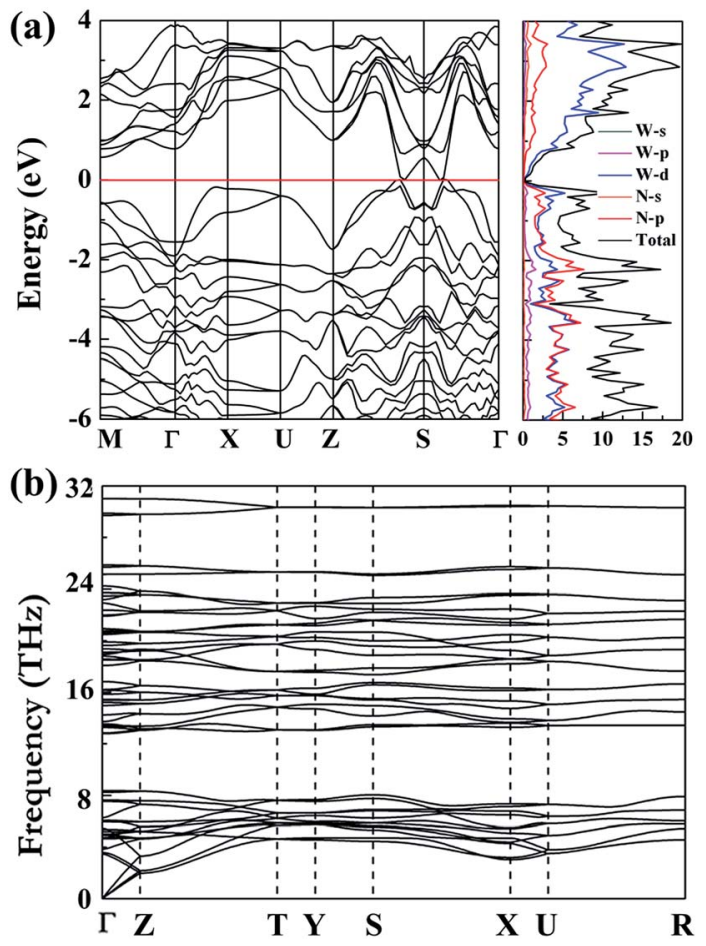

Fig. 4 Calculated band, dos (a) and phonon dispersion curves (b) of the $\mathrm{CmC2}_{1}-\mathrm{WN}_{2}$ at the atmospheric conditions.

The bulk moduli $(B)$ of $C m c 2_{1}-\mathrm{WN}_{2}(437 \mathrm{GPa})$ is larger than the other two structures, which is comparable to the experimental value of diamond of $446 \mathrm{GPa}$ (ref. 43) and indicate its ultra-incompressible nature. The ratio of $B$ and shear modulus $G$ for these structures are found lower than 1.75 (threshold for a material becoming ductile), indicating that these three $\mathrm{WN}_{2}$ compounds are brittle material. In addition, the calculated Young's modulus for novel $C m c 2_{1}-\mathrm{WN}_{2}$ is much lower than that of other two phases. The Vickers hardness of the $C m c 2_{1}-\mathrm{WN}_{2}$ was calculated both using a semiempirical model designed for covalency dominant crystals in particular transition-metal carbides and nitrides (eqn (1) ${ }^{38}$ and a theoretical model which establishes a robust correlation between hardness and elasticity (eqn (2)), ${ }^{39}$

$$
\begin{gathered}
H_{\mathrm{v}}(\mathrm{GPa})=1051 \mathrm{~N}_{\mathrm{e}}^{2 / 3} d^{-2.5} \mathrm{e}^{-1.191 f_{\mathrm{i}}^{-32.2} f_{\mathrm{m}}^{0.55}} \\
H_{\mathrm{v}}=2\left(k^{2} G\right)^{0.585}-3
\end{gathered}
$$

In eqn (1), $N_{\mathrm{e}}$ is the valence electron density $\left(\AA^{-3}\right), d$ is the bond length, $f_{\mathrm{i}}$ is the ionicity of the chemical bond in the crystal on

Table 1 The theoretical elastic constants $C_{i j}(\mathrm{GPa})$, bulk modulus $B(\mathrm{GPa})$, shear modulus $G(\mathrm{GPa})$ Young's modulus $Y$ (GPa), and hardness (GPa) of $\mathrm{Cmc2}_{1}, P 6_{3} / \mathrm{mmc}$ and $\mathrm{P} 4 / \mathrm{mbm}$ are given at atmospheric pressure

\begin{tabular}{lcccccccccccc}
\hline & $C_{11}$ & $C_{12}$ & $C_{13}$ & $C_{23}$ & $C_{33}$ & $C_{44}$ & $C_{66}$ & $B$ & $G$ & $Y$ & $H_{\mathrm{v}}$ tian & $H_{\mathrm{v}}{ }^{\text {chen }}$ \\
\hline $\mathrm{Cmc2}_{1}$ & 641 & 311 & 298 & 232 & 728 & 298 & 251 & 437 & 252 & 596 & 21.9 \\
$P 6_{3} / \mathrm{mmc}^{10}$ & 579 & 195 & 211 & & 973 & 233 & & 364 & 228 & 695 & 23.6 \\
$P 4 / \mathrm{mbm}^{11}$ & 785 & 188 & 165 & & 894 & 275 & 308 & 389 & 300 & 716 & 24.7 \\
& & & & & & & &
\end{tabular}


the Phillips scale, and $f_{\mathrm{m}}$ is a factor of metallicity. In eqn (2), $k$ is the Pugh's ratios $(k=G / \mathrm{B})$. The calculated Vickers hardness (ambient pressure values) for the $C m c 2_{1}-\mathrm{WN}_{2}$ structures is $21.9 \mathrm{GPa}$ and $23.6 \mathrm{GPa}$, respectively indicates that it is a hard material (20 GPa). The hardness of the other two structures are also listed for comparison. ${ }^{10,11}$

Furthermore, we have investigated the electronic properties of the predicted structure. The band structure of $C m c 2_{1}-\mathrm{WN}_{2}$ is plotted in Fig. 4(a) together with the corresponding partial density of states (PDOS). It is found that the $C m c 2_{1}-\mathrm{WN}_{2}$ structure is shown to be metallic with the majority of the DOS near the Fermi level contributed by p-states of nitrogen and d-states of tungsten. Strong hybridization between the W-d and N-p orbitals suggests the strong covalent bonding between the $\mathrm{W}$ and $\mathrm{N}$ atoms, in satisfactory agreement with the ELF analysis. The pronounced peaks in the DOS of $C m c 2_{1}-\mathrm{WN}_{2}$ correspond to flat bands in the band structure. Phonon calculation played a key role in understanding the dynamic stabilities of the predicted structures. The calculations on phonon dispersion clearly show that there is no imaginary phonon frequency in the whole Brillouin zones of $C m c 2_{1}-\mathrm{WN}_{2}$ [Fig. 4(b)], indicating this structure is dynamically stable at ambient pressure that thus suggest the predicted structure could in principle be experimentally synthesized and quenched recovered to atmospheric conditions. We have also investigated the $P 6_{3} / \mathrm{mmc}$ and $P 4 / \mathrm{mbm}$ as well, and the results reveal the both structures are dynamically stable. ${ }^{\mathbf{1 0 , 1 1}}$

\section{Conclusion}

In summary, we have performed the simulations on the thermodynamically stable structures of $\mathrm{WN}_{2}$ over a wide range of pressures combining the first-principles calculations with unbiased structural searching method. We uncovered a novel orthorhombic structure with a space group $C m c 2_{1}$ as the thermodynamically most stable phase for $\mathrm{WN}_{2}$ between 46$113 \mathrm{GPa}$. A comprehensive study of the elastic properties, ELF and hardness demonstrated that the novel $C m c 2_{1}-\mathrm{WN}_{2}$ exhibit excellent mechanical and ultra-incompressible properties. No imaginary phonon frequencies indicate that the structure is dynamically stable therefore it can be prepared at high-pressure and high-temperature and quenched recovered to atmospheric conditions. These findings will inevitably stimulate extensive experimental works on synthesizing these technologically important materials and theoretical work to explore the rich and complex phases of transition-metal and light-element compounds.

\section{Conflicts of interest}

There are no conflicts to declare.

\section{Acknowledgements}

This work is supported by the Natural Science Foundation of China under No. 11747049, Shandong Provincial Natural Science Foundation of China under Grant No. ZR2017LA008,
Science Foundation of Binzhou University under Grant No. 2016 Y03.

\section{References}

1 S. Anwar, Surf. Eng., 2017, 33, 276.

2 P. Nayak, S. Anwar, S. Bajpai and S. Anwar, in AIP Conference Proceedings, 2017, vol. 1832, p. 080063.

3 N. Schönberg, Acta Chem. Scand., 1954, 8, 204.

4 M. Zhang, Y. Qiu, Y. Han, Y. Guo and F. Cheng, J. Power Sources, 2016, 322, 163.

5 Y. Shen, Y. Mai, D. R. McKenzie, Q. Zhang, W. D. McFall and W. E. McBride, J. Appl. Phys., 2000, 88, 1380.

6 M. J. Mehl, D. Finkenstadt, C. Dane, G. L. Hart and S. Curtarolo, Phys. Rev. B, 2015, 91, 184110.

7 P. Hones, N. Martin, M. Regula and F. Lévy, J. Phys. D: Appl. Phys., 2003, 36, 1023.

8 Y. Dong and J. Li, Chem. Commun., 2015, 51, 572.

9 Q. Li, L. Sha, C. Zhu and Y. Yao, Europhys. Lett., 2017, 118, 46001.

10 H. Wang, Q. Li, Y. Li, Y. Xu, T. Cui, A. R. Oganov and Y. Ma, Phys. Rev. B, 2009, 79, 132109.

11 H. Yan, M. Zhang, Q. Wei and P. Guo, J. Alloys Compd., 2013, 581, 508.

12 Z. Zhao, K. Bao, D. Duan, F. Tian, Y. Huang, H. Yu, Y. Liu, B. Liu and T. Cui, Phys. Chem. Chem. Phys., 2015, 17, 13397.

13 Z. Liu, X. Zhou, D. Gall and S. Khare, Comput. Mater. Sci., 2014, 84, 365.

14 D. Suetin, I. Shein and A. Ivanovskii, Russ. Chem. Rev., 2010, 79, 611.

15 C. Lu, Q. Li, Y. Ma and C. Chen, Phys. Rev. Lett., 2017, 119, 115503.

16 S. Wang, X. Yu, Z. Lin, R. Zhang, D. He, J. Qin, J. Zhu, J. Han, L. Wang, H. Mao, J. Zhang and Y. Zhao, Chem. Mater., 2012, 24, 3023.

17 F. Kawamura, H. Yusa and T. Taniguchi, J. Am. Ceram. Soc., 2018, 101, 949.

18 D. Choi and P. N. Kumta, J. Am. Ceram. Soc., 2007, 90, 3113. 19 A. Zerr, G. Miehe and R. Riedel, Nat. Mater., 2003, 2, 185.

20 E. Gregoryanz, M. Sanloup, J. Badro, G. Fiquet, H. K. Mao and R. J. Hemley, Nat. Mater., 2004, 3, 294.

21 J. Crowhurst, A. Goncharov, B. Sadigh, C. Evans, P. Morrall, J. Ferreira and A. Nelson, Science, 2006, 311, 1275.

22 A. F. Young, C. Sanloup, E. Gregoryanz, S. Scandolo, R. H. Hemley and H. K. Mao, Phys. Rev. Lett., 2006, 96, 155501.

23 Y. Wang, J. Lv, L. Zhu and Y. Ma, Comput. Phys. Commun., 2012, 183, 2063.

24 Y. Wang, J. Lv, L. Zhu and Y. Ma, Phys. Rev. B, 2010, 82, 094116.

25 X. Zhang, Y. Wang, J. Lv, C. Zhu, Q. Li, M. Zhang, Q. Li and Y. Ma, J. Chem. Phys., 2013, 138, 114101.

26 Y. Li, J. Hao, H. Liu, Y. Li and Y. Ma, J. Chem. Phys., 2014, 140, 174712.

27 M. Zhang, H. Liu, Q. Li, B. Gao, Y. Wang, H. Li, C. Chen and Y. Ma, Phys. Rev. Lett., 2015, 114, 015502. 
28 L. Zhang, Y. Wang, J. Lv and Y. Ma, Nat. Rev. Mater., 2017, 2, 17005.

29 L. Zhu, H. Liu, C. J. Pickard, G. Zou and Y. Ma, Nat. Chem., 2014, 6, 644.

30 X. Yong, H. Liu, M. Wu, Y. Yao, J. S. Tse, R. Dia and C. S. Yoo, Proc. Natl. Acad. Sci. U. S. A., 2016, 113, 11110.

31 D. M. Ceperley and B. Alder, Phys. Rev. Lett., 1980, 45, 566.

32 J. Ihm, A. Zunger and M. L. Cohen, J. Phys. C: Solid State Phys., 1979, 12, 4409.

33 G. Kresse and J. Furthmüller, Phys. Rev. B, 1996, 54, 11169.

34 G. Kresse and D. Joubert, Phys. Rev. B, 1999, 59, 1758.

35 H. J. Monkhorst and J. D. Pack, Phys. Rev. B, 1976, 13, 5188.

36 A. Togo, F. Oba and I. Tanaka, Phys. Rev. B, 2008, 78, 134106.
37 R. Hill, Proc. Phys. Soc., London, Sect. A, 1952, 65, 349.

38 X. Guo, L. Li, Z. Liu, D. Yu, J. He, R. Liu, B. Xu, Y. Tian and H. Wang, J. Appl. Phys., 2008, 104, 023503.

39 X. Chen, H. Niu, D. Li and Y. Li, Intermetallics, 2011, 19, 1275.

40 A. D. Becke and K. E. Edgecombe, J. Chem. Phys., 1990, 92, 5397.

41 B. Silvi and A. Savin, Nature, 1994, 371, 683.

42 M. Born and R. D. Misra, On the stability of crystal lattices, Cambridge University Press, 1940, vol. 36, p. 466.

43 F. Occelli, D. L. Farber and R. L. Toullec, Nat. Mater., 2003, 2, 151. 